\title{
Stem Cells in Heart Failure
}

\author{
Gabriela Kania, ${ }^{1}$ Kenneth R. Boheler, ${ }^{2}$ Ulf Landmesser, ${ }^{3}$ and Wojciech Wojakowski ${ }^{4}$ \\ ${ }^{1}$ Cardioimmunology, Cardiovascular Research, Institute of Physiology, University of Zürich, Winterthurerstrasse 190, \\ 8057 Zurich, Switzerland \\ ${ }^{2}$ NIH/NIA/GRC/LCS, 5600 Nathan Shock Drive, Baltimore, MD 21224, USA \\ ${ }^{3}$ Department of Cardiology, University Hospital, Rämistrasse 100, 8091 Zurich, Switzerland \\ ${ }^{4}$ Division of Cardiology, Medical University of Silesia, 40-583 Katowice, Poland
}

Correspondence should be addressed to Gabriela Kania, gabriela.kania@uzh.ch

Received 17 October 2011; Accepted 17 October 2011

Copyright ( $) 2011$ Gabriela Kania et al. This is an open access article distributed under the Creative Commons Attribution License, which permits unrestricted use, distribution, and reproduction in any medium, provided the original work is properly cited.

Heart failure is a leading cause of morbidity and mortality in the developed world. Cardiac injury with a significant loss of cardiomyocytes usually leads to a common final pathway of cardiac pathological remodelling that includes fibrosis, and the development and progression of heart failure. The treatment of chronic heart failure generally relies on strategies designed to target and limit cardiac ischemia, additional tissue damage, pathological cardiac remodelling, or hemodynamic impairment and cardiac dyssynchrony. Moreover, understanding of the pathways that chauffeur pathological remodelling as opposed to myocardial repair and regeneration in the failing heart still remains challenging. The identification of stem cells has, however, opened new prospects for the development of cell-based regenerative medicine [1]. Indeed, the heart may contain a pool of resident or circulation-derived stem cells, but, following injury, these endogenous cells are not sufficiently regenerative to fully repair-damaged myocardium [2]. To overcome this insufficiency, treatments have been proposed with exogenous stem cells or their progeny, which may reduce pathological processes in the damaged heart and improve cardiac tissue regeneration in mice and humans. Despite concerted efforts to treat damaged myocardium through cell transplantation, it remains a matter of debate whether the delivery of stem cells or stem cell progeny contributes principally to new cardiac tissue formation, to the activation of endogenous repair mechanisms, or to the modulation of inflammatory processes [3]. More importantly, stem cell-based therapies have resulted in improved cardiac function, and the development of this line of research represents a new frontier in modern cardiovascular research. In this special issue of Stem Cells International, we have assembled a series of original manuscripts and review articles dealing with this research frontier. The articles describe a variety of novel strategies to obtain cells for cardiac repair or regeneration and discuss current efforts, available tools, and new methods for stem cell-based therapies.

Pluripotent stem cells represent one potential source for stem cell-based therapy in the failing heart [4]; however, this kind of therapy has some serious limitations, ranging from ethical issues in humans to the degree of heterogeneity found in cultures of "purified" embryonic stem cell-derived cardiomyocytes (ESC-CMs). Following injection into heart, previous studies demonstrated that ESC-CMs form grafts that may mediate long-term recovery of global and regional myocardial contractile function following infarction. In this issue, K. R. Boheler et al. [5] specifically addressed the question of developmental state and showed that immature hypoxia-resistant ESC-CMs can be isolated in mass in vitro. The authors suggested that the use of immature proliferating CMs that do not seem to form tumors in vivo may serve as a source of innately hypoxia-resistant CMs useful in the treatment of ischemic cardiac disorders. Such an approach might become a viable strategy for treating human cardiac disease states and injuries in the future; however, several obstacles still need to be resolved, including potential immunological responses, safety, and durable improvement of cardiac function in large animal models. 
In a separate paper, S. Schmitteckert et al. [6] propose the transcription factor Lbxl as new marker of differentiating ESC-CMs. Lbx1 plays a role in the migration of muscle progenitor cells in limb buds and determines neuronal differentiation processes $[7,8]$. Since Lbx1 was largely expressed in differentiating ESC-CMs, Lbx1 might represent a novel tool for the identification of proper cell source to induce the reparative processes in the injured heart. Moreover, this finding may provide a model system of Lbx1 target genes and signaling pathways involved in early heart failure caused by Lbx1 inactivation.

An entirely new vision of stem cell-based therapy was presented by S. Liebau et al. [9]. In this paper, the authors focused on calcium-activated potassium channels (SKCas) as important inducers of stem cell differentiation. SKCas are involved in cardiac pacemaker-cell development from ESCs and morphological shaping of neural stem cells $[10,11]$. SKCas are also important modulators of the cytoskeleton rearrangement [12]. Previously, these authors showed that increased SKCas channel activity resulted in a strong and fast differentiation of pluripotent cells followed by a cellfate determination into the cardiac lineage, mainly with a phenotype of cardiac pacemaker-like cells derived from ESC and iPS cells [13]. Here, this group reported the successful generation and characterization of a murine ESC line overexpressing the subtype 4 of SKCas channels in a doxycycline-dependent manner. Overexpression of SKCas4 was increased in cardiac and pacemaker-like cells suggesting SKCas4 as a unique tool to characterize the differentiation of pluripotent cells into cardiac phenotypes. SKCas channelmediated stem cell differentiation might also be applicable to the human system.

Although substantial efforts have been made to develop therapeutic strategies with stem cells to regenerate injured heart [3], there is increasing evidence that stem cells modulate inflammatory processes in a paracrine fashion more so than through direct cardiac tissue regeneration [14]. Recent findings have also suggested that the poor effectiveness of stem cell-based therapies in heart diseases is a result of nonphysiological microenvironment in affected cardiac tissue $[14,15]$. In particular, inflamed myocardium seems to inhibit the cardio-regenerative capacity of transplanted stem cells, while promoting profibrotic processes. A growing body of evidences suggests that the specific signaling milieu of the affected heart is a key determinant of the fate and function of stem cells in the myocardium [16]. Coupling modulation of the myocardial microenvironment with patient-specific stem cells must, therefore, be considered before successful stem cell-based therapies of heart disorders will be achieved. Accordingly, our special issue offers a comprehensive comparison of different sources of stem cells for heart regeneration in basic science and in clinical trials. Moreover, there is a discussion of the potential mechanisms involved in reparative processes [17, 18]. Finally, A. Kleger et al. [19] provided a comprehensive review on the differential and developmental impact of lysophospholipids on cardiovascular development, which represents a novel approach in the field and may have relevance for the niche environment.
Taken together, the compilation of articles in this special issue of Stem Cells International, discusses the current state of stem cell-based therapies. The authors address both experimental and clinical aspects of stem cell research aimed at improving the reparative processes in the failing heart. The three research articles specifically provide novel information designed either to select for specific types of stem cells or to induce the differentiation of pluripotent cells into the phenotype of cardiac lineages. The reviews also offer a broad-based view of current efforts designed to understand the response of stem cells in a "niche" environment or in response to specific molecules. We hope that this issue will be helpful and interesting for basic researchers as well as for clinicians interested in or performing experiments designed to address relevant cardiac issues in regenerative medicine.

\section{Gabriela Kania \\ Kenneth R. Boheler Ulf Landmesser \\ Wojciech Wojakowski}

\section{References}

[1] P. Anversa, J. Kajstura, and A. Leri, "If I can stop one heart from breaking," Circulation, vol. 115, no. 7, pp. 829-832, 2007.

[2] C. E. Murry, M. H. Soonpaa, H. Reinecke et al., "Haematopoietic stem cells do not transdifferentiate into cardiac myocytes in myocardial infarcts," Nature, vol. 428, no. 6983, pp. 664668, 2004.

[3] M. A. Laflamme and C. E. Murry, "Regenerating the heart," Nature Biotechnology, vol. 23, no. 7, pp. 845-856, 2005.

[4] K. R. Boheler, "Pluripotency of human embryonic and induced pluripotent stem cells for cardiac and vascular regeneration," Thrombosis and Haemostasis, vol. 104, no. 1, pp. 2329, 2010.

[5] K. R. Boheler, R. N. Joodi, H. Qiao et al., "Embryonic stem cell-derived cardiomyocyte heterogeneity and the isolation of immature and committed cells for cardiac remodeling and regeneration," Stem Cells International, vol. 2011, Article ID 214203, 10 pages, 2011.

[6] S. Schmitteckert, C. Ziegler, L. Kartes, and A. Rolletschek, "Transcription factor lbxl expression in mouse embryonic stem cell-derived phenotypes," Stem Cells International, vol. 2011, Article ID 130970, 7 pages, 2011.

[7] H. Brohmann, K. Jagla, and C. Birchmeier, "The role of Lbx1 in migration of muscle precursor cells," Development, vol. 127, no. 2, pp. 437-445, 2000.

[8] S. Watanabe, S. Kondo, M. Hayasaka, and K. Hanaoka, "Functional analysis of homeodomain-containing transcription factor Lbx1 in satellite cells of mouse skeletal muscle," Journal of Cell Science, vol. 120, no. 23, pp. 4178-4187, 2007.

[9] S. Liebau, M. Tischendorf, D. Ansorge et al., "An inducible expression system of the calcium-activated potassium channel 4 to study the differential impact on embryonic stem cells," Stem Cells International, vol. 2011, Article ID 456815, 12 pages, 2011.

[10] S. Liebau, B. Vaida, C. Proepper et al., "Formation of cellular projections in neural progenitor cells depends on SK3 channel activity," Journal of Neurochemistry, vol. 101, no. 5, pp. 13381350, 2007. 
[11] S. Liebau, J. Steinestel, L. Linta et al., "An SK3 channel/ nWASP/Abi-1 complex is involved in early neurogenesis," PLoS One, vol. 6, no. 3, Article ID e18148, 2011.

[12] S. J. Kattman, E. D. Adler, and G. M. Keller, "Specification of multipotential cardiovascular progenitor cells during embryonic stem cell differentiation and embryonic development," Trends in Cardiovascular Medicine, vol. 17, no. 7, pp. 240-246, 2007.

[13] A. Kleger, T. Seufferlein, D. Malan et al., "Modulation of calcium-activated potassium channels induces cardiogenesis of pluripotent stem cells and enrichment of pacemaker-like cells," Circulation, vol. 122, no. 18, pp. 1823-1836, 2010.

[14] J. Endo, M. Sano, J. Fujita et al., "Bone marrow-derived cells are involved in the pathogenesis of cardiac hypertrophy in response to pressure overload," Circulation, vol. 116, no. 10, pp. 1176-1184, 2007.

[15] M. Bujak and N. G. Frangogiannis, "The role of TGF- $\beta$ signaling in myocardial infarction and cardiac remodeling," Cardiovascular Research, vol. 74, no. 2, pp. 184-195, 2007.

[16] G. Kania, P. Blyszczuk, and U. Eriksson, "Mechanisms of cardiac fibrosis in inflammatory heart disease," Trends in Cardiovascular Medicine, vol. 19, no. 8, pp. 247-252, 2009.

[17] R. Mingliang, Z. Bo, and W. Zhengguo, "Stem cells for cardiac repair: status, mechanisms, and new strategies," Stem Cells International, vol. 2011, Article ID 310928, 8 pages, 2011.

[18] O. M. Villet, A. Siltanen, T. Pätilä et al., "Advances in cell transplantation therapy for diseased myocardium," Stem Cells International, vol. 2011, Article ID 679171, 8 pages, 2011.

[19] A. Kleger, S. Liebau, Q. Lin, G. von Wichert, and T. Seufferlein, "The impact of bioactive lipids on cardiovascular development," Stem Cells International, vol. 2011, Article ID 916180, 13 pages, 2011. 

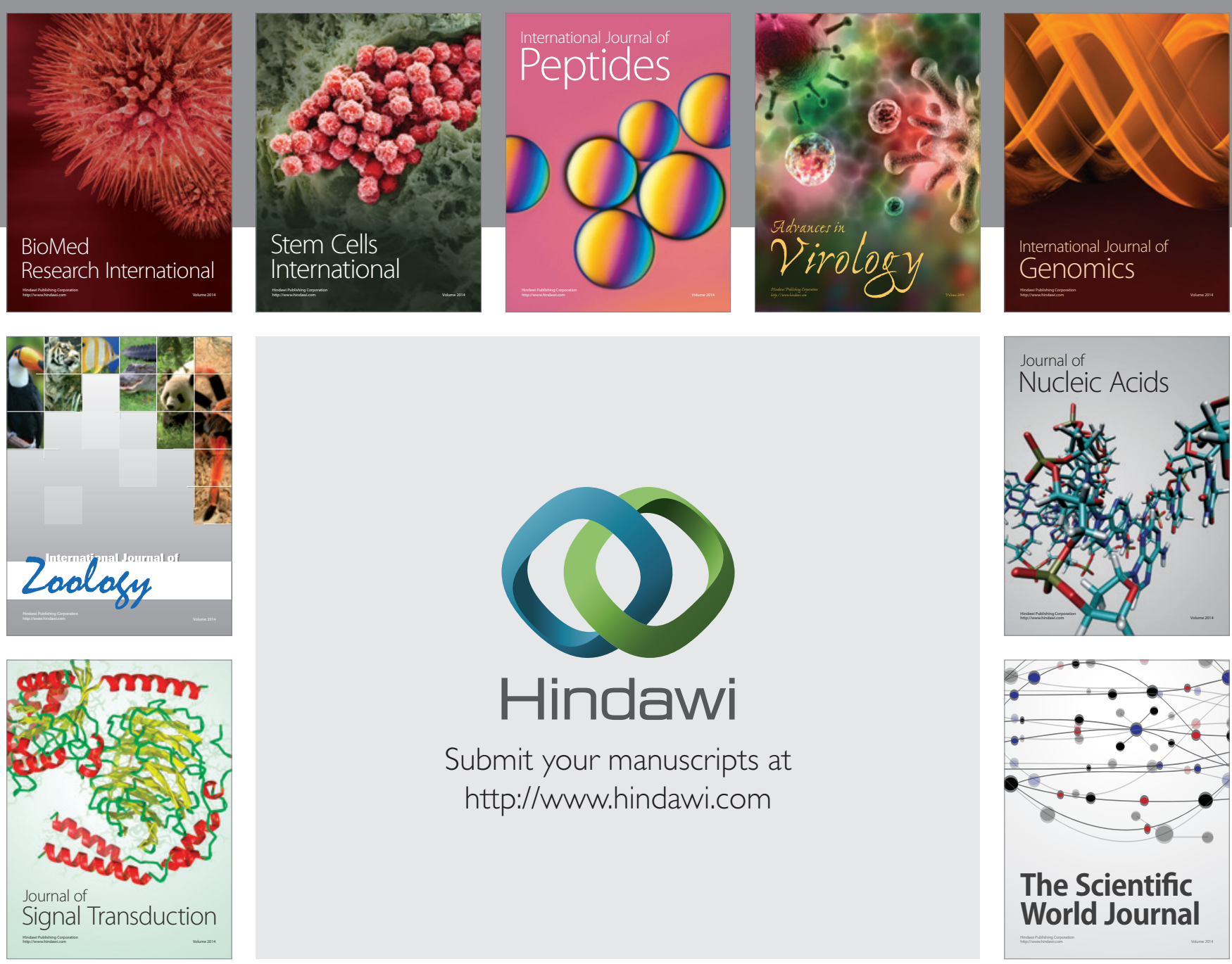

Submit your manuscripts at

http://www.hindawi.com
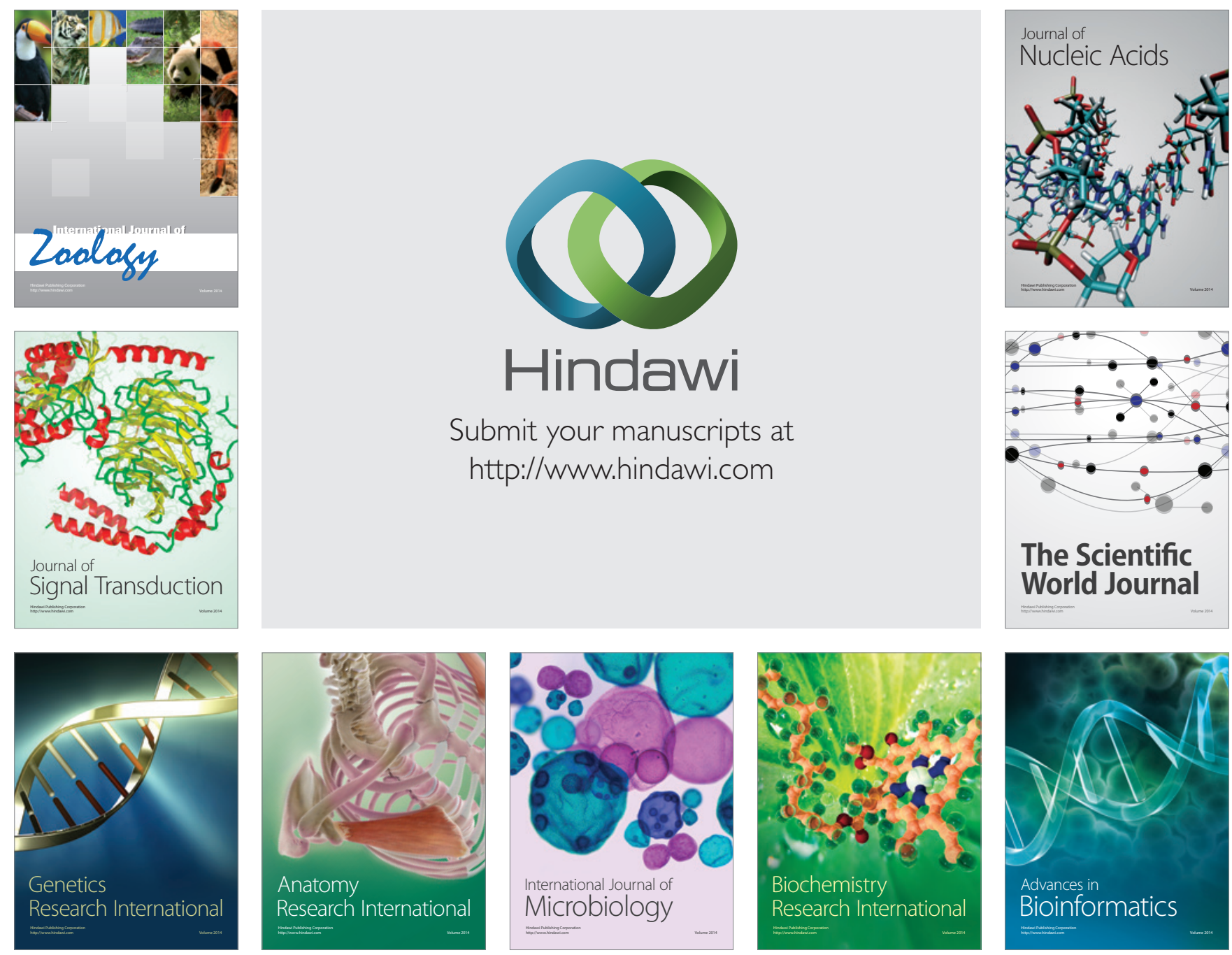

The Scientific World Journal
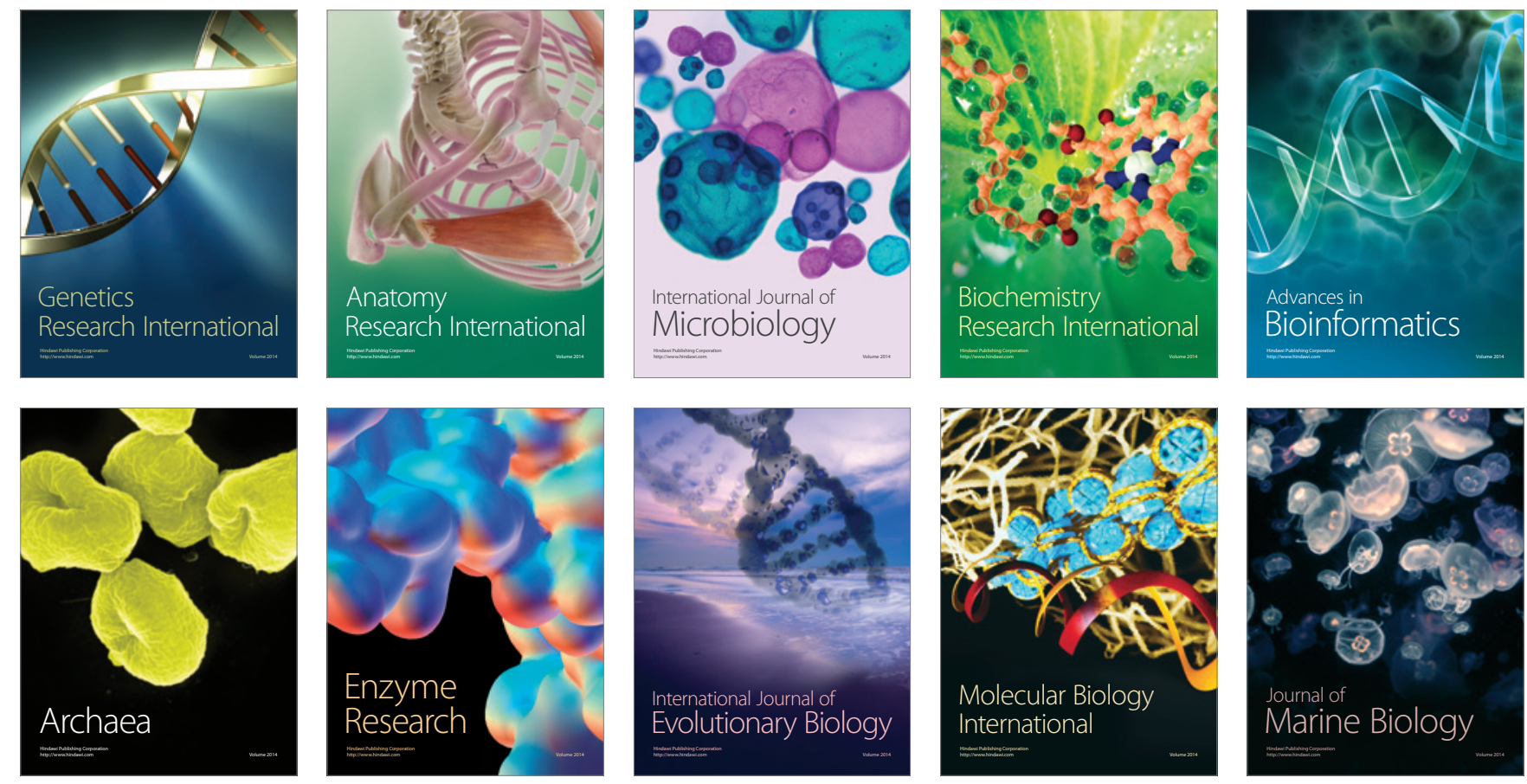